\title{
CHANGE DETECTION WITHIN THE PROCESSING OF THE TANDEM-X CHANGE DEM
}

\author{
Barbara Schweisshelm, Marie Lachaise, Thomas Fritz \\ Remote Sensing Technology Institute, German Aerospace Center (DLR) \\ Münchner Str. 20, 82234 Oberpfaffenhofen, Germany \\ barbara.schweisshelm@dlr.de
}

\begin{abstract}
Over the last years the TanDEM-X mission acquired data for a second global digital elevation model (DEM) the TanDEM$\mathrm{X}$ Change DEM. This new DEM is temporally independent of the former global TanDEM-X DEM and therefore yields the possibility of change detection. In order to decrease the phase noise level the interferometric processing for the Change DEM has been upgraded. This also allows a more accurate change detection. Currently, the processing of the global data is performed operationally. It includes the detection of terrain changes and first examples of detected terrain changes can be presented.
\end{abstract}

Index Terms - TanDEM-X mission, Change DEM, interferometric processing, terrain changes

\section{INTRODUCTION}

The TanDEM-X mission flies two satellites in bistatic formation in order to create digital elevation models. Since the first global TanDEM-X DEM was a great success, it was decided to acquire further data for a second global DEM with the remaining resources [1]. The data for this second DEM, the so-called TanDEM-X Change DEM was acquired between 2017 and 2020. Because of the temporal independence with the former global TanDEM-X DEM (2010 - 2015) it provides the possibility of detecting terrain changes. An updated processing, including a scenewise calibration and adaptive filtering allows an even more accurate change detection. With these adjustments of the Integrated TanDEM-X Processor (ITP) the new data is currently processed at rates up to more than 1000 ChangeRawDEM scenes per day. The status of the production is given in [2].

This paper gives an overview of the interferometric processing and the possibilities as well as limitations of terrain change detection during the operational processing of the TanDEM-X Change DEM.

\section{GENERATION AND INTERFEROMETRIC PROCESSING OF THE CHANGE DEM}

For considerable areas of the Earth the acquisitions for the Change DEM include only one coverage of data. For this reason the dual-baseline approach [3] used for the generation of the global TanDEM-X DEM is no longer feasible and the so-called 'delta-phase' algorithm was developed for the ITP [4]. The 'delta-phase' algorithm makes use of an edited reference DEM. There are different edited DEMs available which will be used for processing the Change DEM data. One edited DEM is specialised on Antarctica an Greenland. It uses the $12 \mathrm{~m}$ posting TanDEM-X DEM [5]. The second edited DEM covers the rest of the world and uses the $30 \mathrm{~m}$ posting TanDEM-X DEM [6]. The third edited DEM is the global WorldDEM30 provided by Airbus D\&S [7]. The operational processing with this DEM started in November 2020. All statistics and images in this paper refer to this processing. A simulated phase of the edited reference DEM instead of a flat Earth equivalent is subtracted from the interferometric phase. The result is a much flatter phase with a low fringe frequency. This reduces the time as well as the error rate of the phase unwrapping immensely [4]. It has to be emphasised that the resulting height values are independent of the height values of the reference DEM.

\subsection{Scenewise Calibration}

The reference DEM does not only simplify the phase unwrapping but is also used to calibrate the processed scenes individually. Therefore, the unwrapped phase difference between the new interferometric phase and the simulated phase from the reference DEM is used. A histogram of a resampled version of the this delta-phase is formed and yields the first estimate of the offset. It is assumed, that the main part of the scene has not changed and therefore the highest peak corresponds to the offset. Furthermore, terrain changes tend to have a broader distribution and appear in lower peaks. The delta-phase pixels within a threshold from the first offset are assumed to correspond to the non changed areas. These pixels are fitted with a plane in order to estimate the offset and a tilt in azimuth up to a certain limit. 


\subsection{Adaptive Filtering}

Additionally, an adaptive filtering approach was developed for the change DEM to make up for the lack of multiple coverages [8]. This approach analyses each scene with respect to azimuth ambiguities. Dependent on their strength the spectral filtering for scenes with low influence of azimuth ambiguities can be reduced. By doing so the phase noise is reduced and the geometric resolution improves. Because the geometric resolution of the Change DEM is supposed to be the same as for the global TanDEM-X DEM [9], the multilooking of the interferogram is adjusted to gain the same geometric resolution while reducing the phase noise even further. This approach is called adaptive because it is adapted for every scene depending on the strength of the azimuth ambiguities. For strong azimuth ambiguities the spectral filter in azimuth direction is kept strong with a coefficient of 0.6 as for the global TanDEM-X DEM. For weaker ambiguities the coefficient can change to either 0.7 or 0.8 and therefore a weaker filter. A distribution of the azimuth spectral filtering coefficient in the current processing is shown in Fig. 1. The influence of the azimuth ambiguities for most scenes is low (0.8) and the full potential of the adaptive filtering is used.

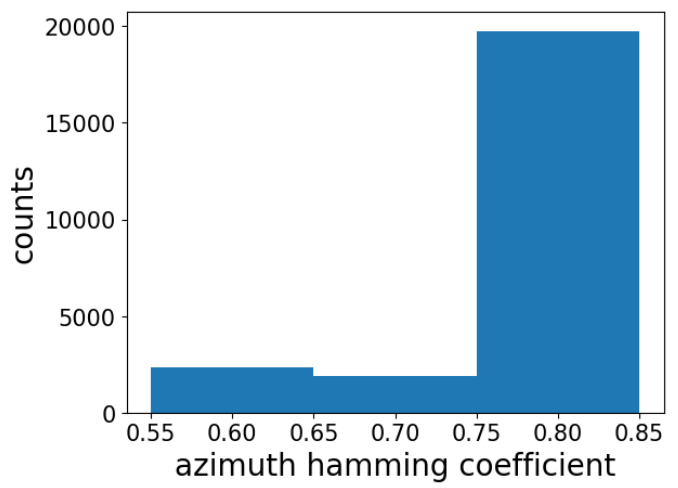

Fig. 1. The distribution of the coefficient of the azimuth spectral hamming filter within the operational Change DEM processing. A subsample of of 24026 scenes was analysed

\section{DEMONSTRATION TERRAIN CHANGES}

During the operational processing of TanDEM-X Change DEM a qualitative change detection is performed. It includes the computation of a delta-height, a visual representation of the height differences between the reference DEM and the new Change DEM heights. It is computed from the phase difference during the processing. Furthermore, there is a Change indication mask (CHM), a geocoded flag mask, which is based on a detailed analysis of the input and reference data. It indicates where the ITP found discrepancies and whether these are reliable and significant. The whole content of this mask would exceed the scope of the paper. Therefore,

the following description is incomplete but sufficient in this context. Detected changes are subdivided into significant and non-significant. For a change to be significant, the height difference has to exceed a threshold (typically $6 \mathrm{~m}$ ) on a regional scale. The changed region has to have a minimum size in order to exclude noisy pixels. Detected changes are also subdivided into reliable and non-reliable changes. This classification depends on the reference DEM used, e.g. pixels which are filled with non TanDEM-X data are counted as non reliable. These two classifications give four different change categories as shown in Table 1. The following examples show the ability of the ITP to detect significant and reliable changes during the ongoing operational processing.

\begin{tabular}{c|c} 
significant \& reliable & significant \& non reliable \\
\hline non significant \& reliable & non significant \& non reliable
\end{tabular}

Table 1. Simplified extract from the color map of the CHM.

\subsection{Small Scale Changes - Mining}

The area shown in Fig. 2 is an acquisition taken over a mine in the North of Australia in September 2017. The DEM shows a typical mining structure. The CHM of this area reveals that there are significant and reliable changes in the area, indicated by the green color (cp. Table 1). The delta height reveals the change directions and magnitudes. In this case it shows that this mine is being closed because the hole gained height (red) and the hill lost height (blue) compared to the former DEM.

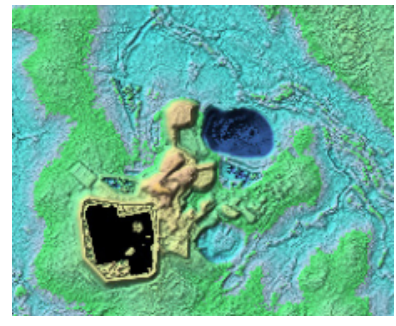

(a) DEM

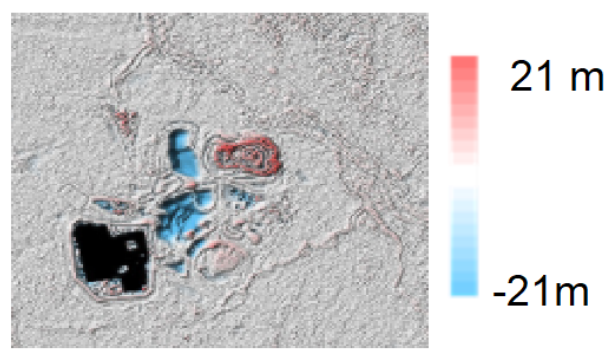

(c) Delta-height

Fig. 2. The DEM reveals a mine structure in North Australia. CHM and delta-height confirm the changes in comparison to the reference. The data was acquired in September 2017. 


\subsection{Large Scale Changes - Forestation and Deforestation}

Another example for terrain changes on a larger scale is possible forestation and deforestation. The area shown in Fig. 3 was recorded in October 2017 over Uruguay. The DEM shows a mountainous forest region. The CHM reveals significant and reliable changes (cp. Table 1). The delta height shows the direction and magnitudes of the changes. It can be seen that parts lost in height (blue probably deforestation) whereas other parts gained high (red probably forestation).

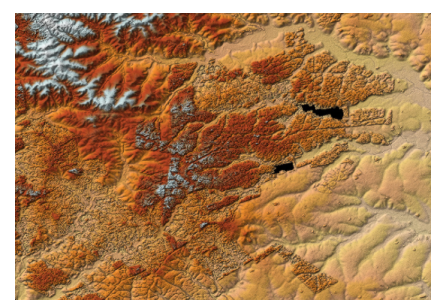

(a) DEM

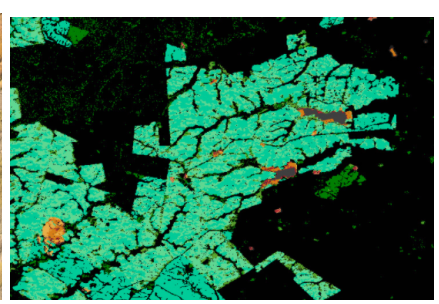

(b) $\mathrm{CHM}$

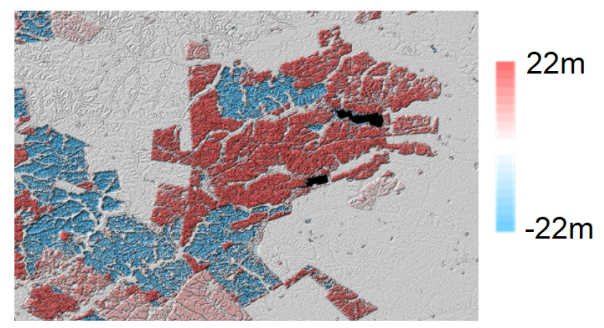

(c) Delta-height

Fig. 3. Terrain changes in Uruguay. The DEM shows a forest area and CHM and delta-height confirm the changes in comparison to the reference DEM. The Change DEM acquisition was taken in October 2017.

\subsection{Statistical Evaluation}

Mining and forestation are only two examples of an abundance of possible terrain changes that are detected. In order to illustrate the number of different detected changes, Fig. 4 shows the distribution of the area per scene which is marked as a significant terrain change.

There are significant changes which are reliable and there are significant changes which are not reliable. Figure 5 illustrates the connection between both. For the ongoing processing Fig. 5 (top) plots the percentage of all significant and reliable changes in negative direction over the total percentage of all significant changes in negative direction. Fig. 5 (bottom) contains the same information for all changes in positive direction. These plots illustrate two things:

- For points close to the red line almost all significant changes are reliable. There is a high number of scenes with only reliable changes in different orders of magnitude.
- The points further away from the red line show that the algorithm is able to distinguish between reliable and non reliable changes. The latter occur mostly in scenes with a small area of changed heights.

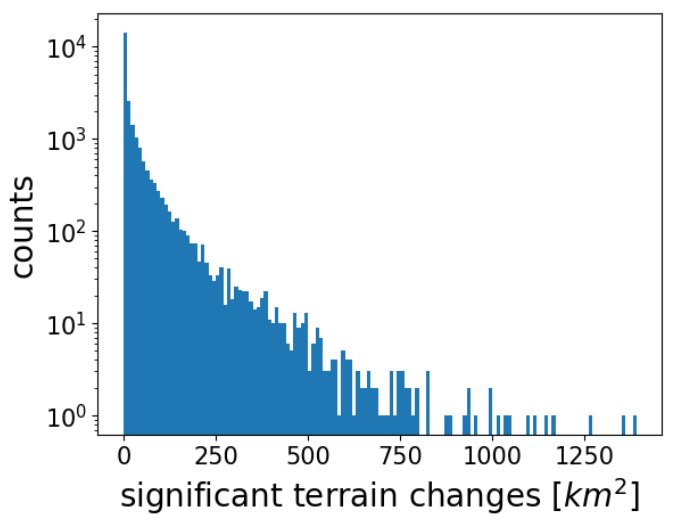

Fig. 4. The distribution of the area $\left[\mathrm{km}^{2}\right]$ that significantly changed per scene (approx. $1500 \mathrm{~km}^{2}$ ) for a subsample of 24026 operationally processed scenes.
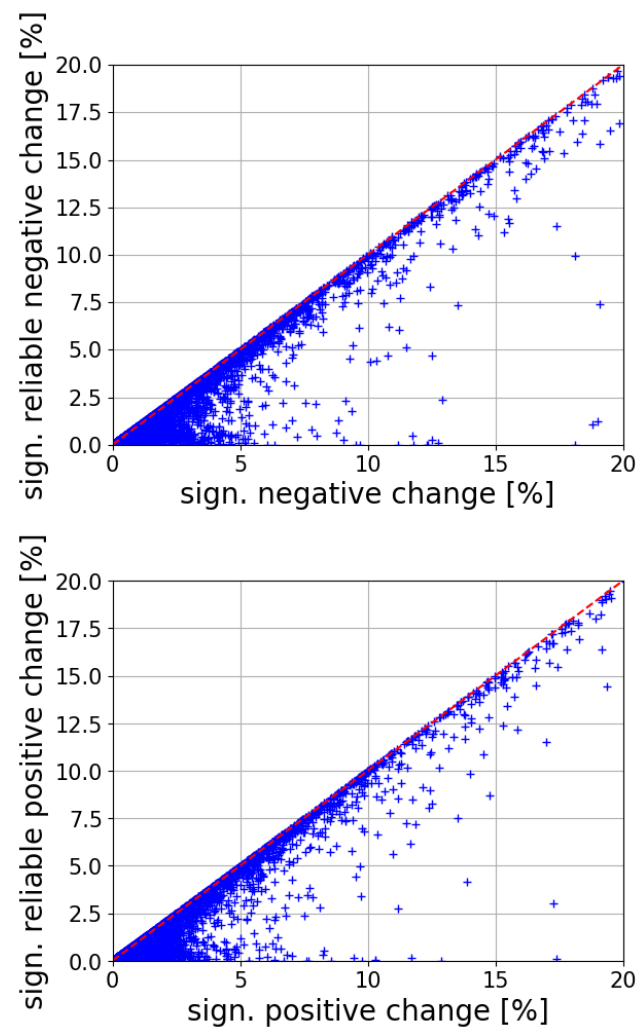

Fig. 5. Connection between significant and reliable and all significant changes in negative (top) and positive (bottom) direction for subsample of 24026 operationally processed scenes. 


\section{LIMITS OF OPERATIONAL CHANGE DETECTION}

The previous section showed that the TanDEM-X Change DEM yields major insights in qualitative terrain changes. However, there are certain limits for the direct change detection within the operational ITP. Two dominant error sources are explained in the following.

\subsection{Reference DEM}

The delta height given as a byproduct of the Change DEM processing refers to the difference between new height data and the specific reference DEM used for the corresponding processing. The processing of the Change DEM data takes place with three different edited versions of the global TanDEM-X DEM. These reference DEMs are edited in different ways, but they have commonalities. As an example, all of them have to fill voids with foreign data which will lead to non comparable data sets within this image. Edited areas can either lead to wrongly denoted changes directly or lead to PU errors within the ITP and therefore the Change DEM.

\subsection{Calibration}

The calibration of each scene as explained in section 2.1 works well in approximately $99 \%$ of the cases. However, there are scenes in which the area of real terrain changes makes up the main area of the scene. If additionally the changes are very uniform, the scene can be wrongly calibrated on the changed area and the detected changes are not real. In general terms, the calibration of Change DEM scenes in the ITP is relative to the reference DEM and assumes only local changes and not absolute changes of entire scenes. Even if there are strong indications for terrain changes, a sophisticated calibration should be performed before a quantitative analysis.

\section{CONCLUSION}

This paper aims to explain the on-going processing of the TanDEM-X Change DEM. Furthermore, it demonstrates possibilities for the characterisation of terrain changes during processing. It could be shown that a qualitative detection of significant changes between the TanDEM-X Change DEM and the edited reference DEM is performed during the operational processing. Even though there are limits of the operational direct change detection, it shows the great potential of quantitative change detection with the TanDEM-X DEM Change DEM in the future.

\section{REFERENCES}

[1] S. Buckreuss, "TerraSAR-X and TanDEM-X Mission Status," European Conference on Synthetic Apperature Radar (EUSAR), pp. 1-4, 2018.

[2] M. Lachaise, M. Bachmann, B. Schweisshelm, and T. Fritz, "The tandem-x change dem: Status of the change raw dems production," in 2021 IEEE International Geoscience and Remote Sensing Symposium (IGARSS), 2021.

[3] M. Lachaise, T. Fritz, and R. Bamler, "The dualbaseline phase unwrapping correction framework for the TanDEM-X mission part 1: Theoretical description and algorithms," 2017, vol. 56, pp. 780-798, IEEE.

[4] M. Lachaise, B. Schweisshelm, and T. Tritz, "The new TanDEM-X Change DEM: Specifications and Interferometric Processing," in Latin American GRSS \& ISPRS Remote Sensing Conference (LAGIRS), 2020, pp. 646651.

[5] M. Huber, B. Wessel, A. Wendleder, J. Hoffmann, and A. Roth, "A framework for an automatical editing of tandem-x digital elevation models," in 2015 IEEE International Geoscience and Remote Sensing Symposium (IGARSS). IEEE, 2015, pp. 3826-3829.

[6] C. González, M. Bachmann, J. Bueso-Bello, P. Rizzoli, and M. Zink, "A fully automatic algorithm for editing the tandem-x global dem," Remote Sensing, vol. 12, no. 23, 2020 .

[7] Airbus Defence and Space GmbH, WorldDEM ${ }^{T M}$ : Technical Product Specification, 2.5 edition, 2019.

[8] B. Schweisshelm, M. Lachaise, and T. Fritz, "An adaptive filtering approach for the new tandem-x change dem," in 2020 IEEE International Geoscience and Remote Sensing Symposium (IGARSS). IEEE, 2020.

[9] B. Wessel et al., TanDEM-X Ground Segment DEM Products Specification Document, Public Document TDGS-PS-0021, issue 3.1 edition, 2016, Available: https://tandemxscience.dlr.de/. 